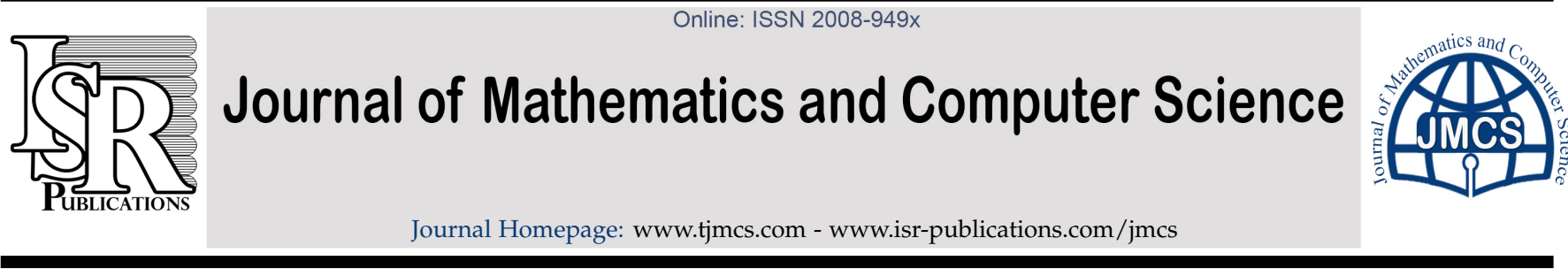

\title{
Characterizations of upper and lower $\alpha\left(\mu_{X}, \mu_{Y}\right)$-continuous multifunctions
}

\author{
Napassanan Srisarakham, Chawalit Boonpok* \\ Mathematics and Applied Mathematics Research Unit, Department of Mathematics, Faculty of Science, Mahasarakham University, \\ Mahasarakham, Thailand.
}

\begin{abstract}
A new class of multifunctions, called upper (lower) $\alpha\left(\mu_{X}, \mu_{Y}\right)$-continuous multifunctions, has been defined and studied. Some characterizations and several properties concerning upper (lower) $\alpha\left(\mu_{X}, \mu_{Y}\right)$-continuous multifunctions are obtained. (C)2017 All rights reserved.
\end{abstract}

Keywords: Generalized topological space, $\mu$ - $\alpha$-open, upper $\alpha\left(\mu_{X}, \mu_{Y}\right)$-continuous multifunction, lower $\alpha\left(\mu_{X}, \mu_{Y}\right)$-continuous multifunction.

2010 MSC: 54A05, 54C08.

\section{Introduction}

General topology has shown its fruitfulness in both the pure and applied directions. In reality it is used in data mining, computational topology for geometric design and molecular design, computer-aided design, computer-aided geometric design, digital topology, information system, non-commutative geometry and its application to particle physics, one can observe the influence made in these realms of applied research by general topological spaces, properties and structures. Continuity is a basic concept for the study of generalized topological spaces. This concept has been extended to the setting of multifunctions and has been generalized by weaker forms of open sets such as $\alpha$-open sets [18], semi-open sets [15], preopen sets [16], $\beta$-open sets [1] and semi-preopen sets [3]. Multifunctions and of course continuous multifunctions stand among the most important and most researched points in the whole of the mathematical science. Many different forms of continuous multifunctions have been introduced over the years. Some of them are semi-continuity [22], $\alpha$-continuity [17], precontinuity [24], quasi-continuity [23], $\gamma$-continuity [2] and $\delta$-precontinuity [21]. Most of these weaker forms of continuity, in ordinary topology such as $\alpha$-continuity and $\beta$-continuity, have been extended to multifunctions [20,25-28]. Császár [5] introduced the notions of generalized topological spaces and generalized neighbourhood systems. The classes of topological spaces and neighbourhood systems are contained in these classes, respectively. Specifically, he introduced the notions of continuous functions on generalized topological spaces and investigated the characterizations of

\footnotetext{
*Corresponding author

Email address: chawalit.b@msu.ac.th (Chawalit Boonpok)
}

doi:10.22436/jmcs.017.02.07 
generalized continuous functions. Kanibir and Reilly [14] extended these concepts to multifunctions. The purpose of the present paper is to define the notion of upper (lower) $\alpha\left(\mu_{X}, \mu_{Y}\right)$-continuous multifunctions and to obtain several characterizations and several properties of these multifunctions.

\section{Preliminaries}

Let $X$ be a set and denote $\mathcal{P}(X)$ the power set of $X$. We call a class $\mu \subseteq \mathcal{P}(X)$ a generalized topology (briefly GT) on $X$, if $\emptyset \in \mu$ and an arbitrary union of elements of $\mu$ belongs to $\mu$ [5]. A set with a GT is said to be a generalized topological space (briefly GTS). For a GTS $(X, \mu)$, the elements of $\mu$ are called $\mu$-open sets and the complements of $\mu$-open sets are called $\mu$-closed sets. For $A \subseteq X$, we denote by $c_{\mu}(A)$ the intersection of all $\mu$-closed sets containing $A$ and by $i_{\mu}(A)$ the union of all $\mu$-open sets contained in A. Then we have $i_{\mu}\left(i_{\mu}(A)\right)=i_{\mu}(A), c_{\mu}\left(c_{\mu}(A)\right)=c_{\mu}(A)$ and $i_{\mu}(A)=X-c_{\mu}(X-A)$. According to [11], for $A \subseteq X$ and $x \in X$, we have $x \in c_{\mu} A$ iff $x \in M \in \mu$ implies $M \cap A \neq \emptyset$. Let $\mathcal{B} \subseteq \mathcal{P}(X)$ satisfy $\emptyset \in \mathcal{B}$. Then all unions of some elements of $\mathcal{B}$ constitute a GT $\mu(\mathcal{B})$ and $\mathcal{B}$ is said to be a base for $\mu(\mathcal{B})$ [10]. Let $\mu$ be a GT on a set $X \neq \emptyset$. Observe that $X \in \mu$ must not hold, if all the same $X \in \mu$ then we say that the GT $\mu$ is strong [7]. In general, let $M_{\mu}$ denote the union of all elements of $\mu$, of course, $M_{\mu} \in \mu$ and $M_{\mu}=X$, iff $\mu$ is a strong GT. Let us now consider those GT's $\mu$ that satisfy the condition: if $M, M^{\prime} \in \mu$ then $M \cap M^{\prime} \in \mu$. We shall call such a GT quasi-topology (briefly QT) [9], the QT's clearly are very near to the topologies.

A subset $A$ of a generalized topological space $(X, \mu)$ is said to be $\mu$-semi-open [8] (resp. $\mu$-preopen, $\mu$ - $\alpha$-open, $\mu$ - $\beta$-open), if $A \subseteq c_{\mu}\left(i_{\mu}(A)\right)$ (resp. $A \subseteq i_{\mu}\left(c_{\mu}(A)\right), A \subseteq i_{\mu}\left(c_{\mu}\left(i_{\mu}(A)\right)\right), A \subseteq c_{\mu}\left(i_{\mu}\left(c_{\mu}(A)\right)\right)$ ). The family of all $\mu$-semi-open (resp. $\mu$-preopen, $\mu$ - $\alpha$-open, $\mu$ - $\beta$-open) sets of $X$ containing a point $x \in X$ is denoted by $\sigma(\mu, x)$ (resp. $\pi(\mu, x), \alpha(\mu, x), \beta(\mu, x)$ ). The family of all $\mu$-semi-open (resp. $\mu$-preopen, $\mu$ - $\alpha$-open, $\mu$ - $\beta$-open) sets of $X$ is denoted by $\sigma(\mu)$ (resp. $\pi(\mu), \alpha(\mu), \beta(\mu)$ ). It is shown in [8, Lemma 2.1] that $\alpha(\mu)=\sigma(\mu) \cap \pi(\mu)$ and it is obvious that $\sigma(\mu) \cup \pi(\mu) \subseteq \beta(\mu)$. The complement of a $\mu$-semi-open (resp. $\mu$-preopen, $\mu$ - $\alpha$-open, $\mu$ - $\beta$-open) set is said to be $\mu$-semi-closed (resp. $\mu$-preclosed, $\mu$ - $\alpha$-closed, $\mu$ - $\beta$-closed).

The intersection of all $\mu$-semi-closed (resp. $\mu$-preclosed, $\mu$ - $\alpha$-closed, $\mu$ - $\beta$-closed) sets of $X$ containing $A$ is denoted by $c_{\sigma}(A) . c_{\pi}(A), c_{\alpha}(A)$ and $c_{\beta}(A)$ are defined similarly. The union of all $\mu$-semi-open (resp. $\mu$-preopen, $\mu$ - $\alpha$-open, $\mu$ - $\beta$-open) sets of $X$ contained in $A$ is denoted by $i_{\sigma}(A), i_{\pi}(A), i_{\alpha}(A)$ and $i_{\beta}(A)$, respectively.

Now let $K \neq \emptyset$ be an index set, $X_{k} \neq \emptyset$ for $k \in K$, and $X=\prod_{k \in K} X_{k}$ the Cartesian product of the sets $X_{k}$. We denote by $p_{k}$ the projection $p_{k}: X \rightarrow X_{k}$. Suppose that, for $k \in K, u_{k}$ is a given GT on $X_{k}$. Let us consider all sets of the form $\prod_{k \in K} X_{k}$ where $M_{k} \in \mu_{k}$ and with the exception of a finite number of indices $k, M_{k}=Z_{k}=M_{\mu_{k}}$. We denote by $\mathcal{B}$ the collection of all these sets. Clearly $\emptyset \in \mathcal{B}$ so that we can define a GT $\mu=\mu(\mathcal{B})$ having $\mathcal{B}$ for base. We call $\mu$ the product [12] of the GT's $\mu_{k}$ and denote it by $\mathbf{P}_{\mathrm{k} \in \mathrm{K}} \mu_{\mathrm{k}}$.

Let us write $i=i_{\mu}, c=c_{\mu}, i_{k}=i_{\mu_{k}}, c_{k}=c_{\mu_{k}}$. Consider in the following $A_{k} \subseteq X_{k}, A=\prod_{k \in K} A_{k}$, $x \in \prod_{k \in K} X_{k}$ and $x_{k}=p_{k}(x)$.

Proposition 2.1 ([12]). $c A=\prod_{k \in K} c_{k} A_{k}$.

Proposition 2.2 ([32]). Let $A=\prod_{k \in K} A_{k} \subseteq \prod_{k \in K} X_{k}$ and $K_{0}$ be a finite subset of $K$. If $A_{k} \in\left\{M_{k}, X_{k}\right\}$ for each $k \in \mathrm{K}-\mathrm{K}_{0}$, then $\mathrm{i} A=\prod_{k \in \mathrm{K}} i_{\mathrm{k}} \AA_{\mathrm{k}}$.

Throughout this paper, the spaces $\left(X, \mu_{X}\right)$ and $\left(Y, \mu_{Y}\right)$ (or simply $X$ and $Y$ ) always mean generalized topological spaces. By a multifunction $F: X \rightarrow Y$, we mean a point-to-set correspondence from $X$ into $Y$, and we always assume that $F(x) \neq \emptyset$ for all $x \in X$. For a multifunction $F: X \rightarrow Y$, we shall denote the upper and lower inverse of a set $G$ of $Y$ by $F^{+}(G)$ and $F^{-}(G)$, respectively, that is $F^{+}(G)=\{x \in X: F(x) \subseteq G\}$ and $F^{-}(G)=\{x \in X: F(x) \cap G \neq \emptyset\}$. In particular, $F^{-}(y)=\{x \in X: y \in F(x)\}$ for each point $y \in Y$. For each $A \subseteq X, F(A)=\cup_{x \in A} F(X)$. Then $F$ is said to be a surjection, if $F(X)=Y$ or equivalently, if for each $y \in Y$ there exists an $x \in X$ such that $y \in F(x)$. 
Definition $2.3([4])$. Let $\left(X, \mu_{X}\right)$ and $\left(Y, \mu_{Y}\right)$ be generalized topological spaces. A multifunction $F: X \rightarrow Y$ is said to be:

(1) upper $\beta\left(\mu_{X}, \mu_{Y}\right)$-continuous at a point $x \in X$, if for each $\mu_{Y}$-open set $V$ of $Y$ such that $F(x) \subseteq V$, there exists $\mathrm{U} \in \beta\left(\mu_{X}, x\right)$ such that $\mathrm{F}(\mathrm{U}) \subseteq \mathrm{V}$;

(2) lower $\beta\left(\mu_{X}, \mu_{Y}\right)$-continuous at a point $x \in X$, if for each $\mu_{Y}$-open set $V$ of $Y$ such that $F(x) \cap V \neq \emptyset$, there exists $U \in \beta\left(\mu_{X}, x\right)$ such that $F(z) \cap V \neq \emptyset$, for every $z \in U$;

(3) upper (resp. lower) $\beta\left(\mu_{X}, \mu_{Y}\right)$-continuous, if $F$ has this property at each point of $X$.

\section{Characterizations}

In this section, the notion of upper (lower) $\alpha\left(\mu_{X}, \mu_{Y}\right)$-continuous multifunctions is introduced and some characterizations and basic properties of upper (lower) $\alpha\left(\mu_{X}, \mu_{Y}\right)$-continuous multifunctions are investigated and obtained.

Definition 3.1. Let $\left(X, \mu_{X}\right)$ and $\left(Y, \mu_{Y}\right)$ be generalized topological spaces. A multifunction $F: X \rightarrow Y$ is said to be:

(1) upper $\alpha\left(\mu_{X}, \mu_{Y}\right)$-continuous at a point $x \in X$, if for each $\mu_{Y}$-open set $V$ of $Y$ such that $F(x) \subseteq V$, there exists $\mathrm{U} \in \alpha\left(\mu_{\mathrm{X}}, \mathrm{x}\right)$ such that $\mathrm{F}(\mathrm{U}) \subseteq \mathrm{V}$;

(2) lower $\alpha\left(\mu_{X}, \mu_{Y}\right)$-continuous at a point $x \in X$, if for each $\mu_{Y}$-open set $V$ of $Y$ such that $F(x) \cap V \neq \emptyset$, there exists $\mathrm{U} \in \alpha\left(\mu_{X}, x\right)$ such that $F(z) \cap V \neq \emptyset$, for every $z \in U$;

(3) upper (resp. lower) $\alpha\left(\mu_{X}, \mu_{Y}\right)$-continuous, if $F$ has this property at each point of $X$.

Example 3.2. Let $X=\{1,2,3\}$ and $Y=\{a, b, c\}$. Define a generalized topology $\mu_{X}=\{\emptyset,\{1\},\{1,2\}\}$ on $X$ and a generalized topology $\mu_{Y}=\{\emptyset,\{a\},\{a, b\}\}$ on $Y$. A multifunction $F:\left(X, \mu_{X}\right) \rightarrow\left(Y, \mu_{Y}\right)$ is defined as follows: $F(1)=F(2)=\{a\}$ and $F(3)=\{c\}$. Then $F$ is upper $\alpha\left(\mu_{X}, \mu_{Y}\right)$-continuous.

Remark 3.3. For a multifunction $\mathrm{F}: \mathrm{X} \rightarrow \mathrm{Y}$, the following implication hold:

$$
\text { upper } \alpha\left(\mu_{X}, \mu_{Y}\right) \text {-continuity } \Longrightarrow \text { upper } \beta\left(\mu_{X}, \mu_{Y}\right) \text {-continuity. }
$$

The converse of the implication is not true in general as shown by the following example.

Example 3.4. Let $X=\{1,2,3\}$ and $Y=\{a, b, c\}$. Define a generalized topology

$$
\mu_{X}=\{\emptyset,\{1\},\{2\},\{1,2\}\},
$$

on $X$ and a generalized topology $\mu_{Y}=\{\emptyset,\{a\},\{b\},\{a, b\}\}$ on $Y$. A multifunction $F:\left(X, \mu_{X}\right) \rightarrow\left(Y, \mu_{Y}\right)$ is defined as follows: $F(1)=F(2)=\{b\}$ and $F(3)=\{a\}$. Then $F$ is upper $\beta\left(\mu_{X}, \mu_{Y}\right)$-continuous but not upper $\alpha\left(\mu_{X}, \mu_{Y}\right)$-continuous, since $\{a\}$ is $\mu_{Y}$-open but $F^{+}(\{a\})=\{3\}$ is not $\mu_{X-\alpha \text {-open. }}$

Lemma 3.5. The following are equivalent for a subset $\mathrm{A}$ of a generalized topological space $\left(\mathrm{X}, \mu_{\mathrm{X}}\right)$ :

(1) $A \in \alpha\left(\mu_{X}\right)$;

(2) $\mathrm{U} \subseteq A \subseteq i_{\mu_{X}}\left(c_{\mu_{X}}(\mathrm{U})\right)$ for some $\mu_{\mathrm{X}}$-open set $\mathrm{U}$;

(3) $\mathrm{U} \subseteq \mathrm{A} \subseteq \mathrm{c}_{\sigma_{X}}(\mathrm{U})$ for some $\mu_{X}$-open set $\mathrm{U}$;

(4) $A \subseteq c_{\sigma_{X}}\left(i_{\mu_{X}}(A)\right)$.

Theorem 3.6. Let $\left(X, \mu_{X}\right)$ be a quasi-topological space and $\left(Y, \mu_{Y}\right)$ a generalized topological space. The following are equivalent for a multifunction $\mathrm{F}: \mathrm{X} \rightarrow \mathrm{Y}$ :

(1) $\mathrm{F}$ is upper $\alpha\left(\mu_{X}, \mu_{Y}\right)$-continuous at $x \in X$;

(2) $x \in c_{\sigma_{X}}\left(i_{\mu_{X}}\left(F^{+}(V)\right)\right)$ for any $\mu_{Y-o p e n ~ s e t ~} V$ of $Y$ containing $F(x)$; 


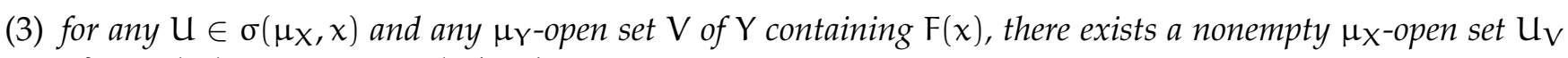
of $\mathrm{X}$ such that $\mathrm{U}_{\mathrm{V}} \subseteq \mathrm{U}$ and $\mathrm{F}\left(\mathrm{U}_{\mathrm{V}}\right) \subseteq \mathrm{V}$.

Proof.

$(1) \Rightarrow(2)$ : Let $V$ be any $\mu_{Y}$-open such that $F(x) \subseteq V$. Then there exists $U \in \alpha\left(\mu_{X}\right)$ containing $x$ such that $\mathrm{F}(\mathrm{U}) \subseteq \mathrm{V}$, hence $\mathrm{x} \in \mathrm{U} \subseteq \mathrm{F}^{+}(\mathrm{V})$. Since $\mathrm{U}$ is $\mu_{\mathrm{X}}$ - $\alpha$-open, by Lemma 3.5 we have $x \in \mathrm{U} \subseteq \mathrm{c}_{\sigma_{X}}\left(i_{\mu_{X}}(\mathrm{U})\right) \subseteq$ $c_{\sigma_{X}}\left(i_{\mu_{X}}\left(F^{+}(V)\right)\right)$.

$(2) \Rightarrow(3)$ : Let $V$ be any $\mu_{Y}$-open set of $Y$ such that $F(x) \subseteq V$. Then $x \in c_{\sigma_{X}}\left(i_{\mu_{X}}\left(F^{+}(V)\right)\right)$. Let $U$ be any $\mu_{X}$-semi-open set containing $x$. Then $U \cap i_{\mu_{X}}\left(F^{+}(V)\right) \neq \emptyset$ and $U \cap i_{\mu_{X}}\left(F^{+}(V)\right) \in \sigma\left(\mu_{X}\right)$. Put $\mathrm{U}_{\mathrm{V}}=i_{\mu_{X}}\left(\mathrm{U} \cap i_{\mu_{X}}\left(F^{+}(V)\right)\right)$, then $U_{V}$ is a nonempty $\mu_{Y^{-}}$open set of $X, U_{V} \subseteq U$ and $F\left(U_{V}\right) \subseteq \mathrm{V}$.

(3) $\Rightarrow(1)$ : Let $V$ be any $\mu_{Y}$-open set of $Y$ containing $F(x)$. For each $U \in \sigma\left(\mu_{X}, x\right)$, there exists a nonempty $\mu_{X}$-open set $U_{V}$ such that $U_{V} \subseteq U$ and $F\left(U_{V}\right) \subseteq V$. Let $W=\cup\left\{U_{V}: U \in \sigma\left(\mu_{X}, x\right)\right\}$. Then $W$ is $\mu_{X}$-open in $X, x \in c_{\sigma_{X}}(W)$ and $F(W) \subseteq V$. Put $S=W \cup\{x\}$, then $W \subseteq S \subseteq c_{\sigma_{X}}(W)$. Therefore, by Lemma 3.5, $x \in S \in \alpha\left(\mu_{X}\right)$ and $F(S) \subseteq V$. This shows that $F$ is upper $\alpha\left(\mu_{X}, \mu_{Y}\right)$ continuous at $x$.

Theorem 3.7. Let $\left(\mathrm{X}, \mu_{\mathrm{X}}\right)$ be a quasi-topological space and $\left(\mathrm{Y}, \mu_{\mathrm{Y}}\right)$ a generalized topological space. The following are equivalent for a multifunction $\mathrm{F}: \mathrm{X} \rightarrow \mathrm{Y}$ :

(1) $\mathrm{F}$ is lower $\alpha\left(\mu_{X}, \mu_{Y}\right)$-continuous at $\mathrm{x} \in \mathrm{X}$;

(2) $x \in c_{\sigma_{X}}\left(i_{\mu_{X}}\left(F^{-}(\mathrm{V})\right)\right)$ for any $\mu_{Y^{-}}$open set $\mathrm{V}$ of $\mathrm{Y}$ such that $\mathrm{F}(\mathrm{x}) \cap \mathrm{V} \neq \emptyset$;

(3) for any $\mathrm{U} \in \sigma\left(\mu_{\mathrm{X}}, \mathrm{x}\right)$ and any $\mu_{\mathrm{Y}}$-open set $\mathrm{V}$ of $\mathrm{Y}$ such that $\mathrm{F}(\mathrm{x}) \cap \mathrm{V} \neq \emptyset$, there exists a nonempty $\mu_{\mathrm{X}-\text {-open }}$ set $\mathrm{U}_{\mathrm{V}}$ of $\mathrm{X}$ such that $\mathrm{F}(\mathrm{u}) \cap \mathrm{V} \neq \emptyset$, for every $\mathrm{u} \in \mathrm{U}_{\mathrm{V}}$ and $\mathrm{U}_{\mathrm{V}} \subseteq \mathrm{V}$.

Proof. The proof is similar to that of Theorem 3.6.

Lemma 3.8. The following are equivalent for a subset $\mathrm{A}$ of a generalized topological space $\left(\mathrm{X}, \mu_{\mathrm{X}}\right)$ :

(1) $A$ is $\mu_{X}-\alpha$-closed in $X$, if and only if $i_{\sigma_{X}}\left(c_{\mu_{X}}(A)\right) \subseteq A$;

(2) $i_{\sigma_{X}}\left(c_{\mu_{X}}(A)\right)=c_{\mu_{X}}\left(i_{\mu_{X}}\left(c_{\mu_{X}}(A)\right)\right)$;

(3) $c_{\alpha_{X}}(A)=A \cup c_{\mu_{X}}\left(i_{\mu_{X}}\left(c_{\mu_{X}}(A)\right)\right)$.

A subset $N_{X}$ of a generalized topological space $\left(X, \mu_{X}\right)$ is said to be $\mu_{X}$-neighbourhood (resp. $\mu_{X}-\alpha-$ neighbourhood) of a point $x \in X$, if there exists a $\mu_{X}$-open (resp. $\mu_{X}-\alpha$-open) set $U$ such that $x \in U \subseteq N_{x}$.

Theorem 3.9. Let $\left(\mathrm{X}, \mu_{\mathrm{X}}\right)$ and $\left(\mathrm{Y}, \mu_{\mathrm{Y}}\right)$ be generalized topological spaces. The following are equivalent for a multifunction $\mathrm{F}: \mathrm{X} \rightarrow \mathrm{Y}$ :

(1) $\mathrm{F}$ is upper $\alpha\left(\mu_{X}, \mu_{Y}\right)$-continuous;

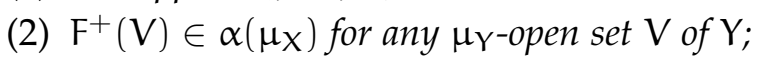

(3) $\mathrm{F}^{-}(\mathrm{M})$ is $\mu_{\mathrm{X}}-\alpha$-closed in $\mathrm{X}$ for any $\mu_{\mathrm{Y}^{-}}$-closed set $\mathrm{M}$ of $\mathrm{Y}$;

(4) $i_{\sigma_{X}}\left(c_{\mu_{X}}\left(F^{-}(B)\right)\right) \subseteq F^{-}\left(c_{\mu_{Y}}(A)\right)$ for any subset $A$ of $Y$;

(5) $c_{\alpha_{X}}\left(F^{-}(A)\right) \subseteq F^{-}\left(c_{\mu_{Y}}(A)\right)$ for any subset $A$ of $Y$;

(6) for each point $\mathrm{x}$ of $\mathrm{X}$ and each $\mu_{\mathrm{Y}}$-neighbourhood $\mathrm{V}$ of $\mathrm{F}(\mathrm{x}), \mathrm{F}^{+}(\mathrm{V})$ is a $\mu_{\mathrm{X}}-\alpha$-neighbourhood of $\mathrm{x}$;

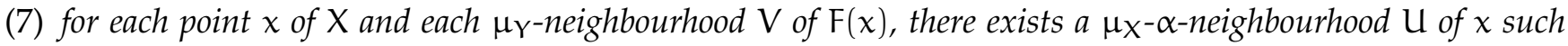
that $\mathrm{F}(\mathrm{U}) \subseteq \mathrm{V}$.

Proof.

$(1) \Rightarrow(2)$ : Let $V$ be any $\mu_{Y}$-open set of $Y$ and let $x \in F^{+}(V)$. By Theorem 3.6, $x \in c_{\sigma_{X}}\left(i_{\mu_{X}}\left(F^{+}(V)\right)\right)$. Therefore, we obtain $F(x) \subseteq c_{\sigma_{X}}\left(i_{\mu_{X}}\left(F^{+}(V)\right)\right)$. It follows from Lemma 3.5 that $F^{+}(V) \in \alpha\left(\mu_{X}\right)$.

$(2) \Leftrightarrow(3)$ : This follows from the fact that $F^{+}(Y-A)=X-F^{-}(A)$ for any subset $A$ of $Y$. 
$(3) \Rightarrow(4)$ : Let $A$ be any subset of $Y$. Then $F^{-}\left(c_{\mu_{Y}}\right)$ is closed in $Y$. By Lemma 3.8, we have $i_{\sigma_{X}}\left(c_{\mu_{X}}\left(F^{-}(A)\right)\right) \subseteq$ $i_{\sigma_{X}}\left(c_{\mu_{X}}\left(F^{-}\left(c_{\mu_{Y}}(A)\right)\right)\right) \subseteq F^{-}\left(c_{\mu_{Y}}(A)\right)$.

$(4) \Rightarrow(5)$ : Let $A$ be any subset of $Y$. By Lemma 3.8, we have

$$
c_{\alpha_{X}}\left(F^{-}(A)\right)=F^{-}(A) \cup i_{\sigma_{X}}\left(c_{\mu_{X}}\left(F^{-}(A)\right)\right) \subseteq F^{-}\left(c_{\mu_{Y}}(A)\right) .
$$

(5) $\Rightarrow$ (3): Let $M$ be any $\mu_{Y}$-closed set of $Y$. Then we have

$$
c_{\alpha_{X}}\left(F^{-}(M)\right) \subseteq F^{-}\left(c_{\mu_{Y}}(M)\right)=F^{-}(M) .
$$

This shows that $F^{-}(M)$ is $\mu_{X-}-\alpha$-closed in $X$.

$(2) \Rightarrow(6)$ : Let $x \in X$ and $V$ be a $\mu_{Y}$-neighbourhood of $F(x)$. Then there exists a $\mu_{Y}$-open set $G$ of $Y$ such that $F(x) \subseteq G \subseteq V$. Therefore, we obtain $x \in F^{+}(G) \subseteq F^{+}(V)$. Since $F^{+}(G) \in \alpha\left(\mu_{X}\right), F^{+}(V)$ is a $\mu_{X}-\alpha$-neighbourhood of $x$.

(6) $\Rightarrow(7)$ : Let $x \in X$ and $V$ be a $\mu_{Y}$-neighbourhood of $F(x)$. Put $U=F^{+}(V)$, then $U$ is a $\mu_{X^{-}} \alpha-$ neighbourhood of $x$ and $F(U) \subseteq V$.

$(7) \Rightarrow(1)$ : Let $x \in X$ and $V$ be any $\mu_{Y}$-open set of $Y$ such that $F(x) \subseteq V$. Then $V$ is a $\mu_{Y}$-neighbourhood of $F(x)$. There exists a $\mu_{X}-\alpha$-neighbourhood $U$ of $x$ such that $F(U) \subseteq V$. Therefore, there exists $W \in \alpha\left(\mu_{X}\right)$ such that $\mathrm{x} \in \mathrm{W} \subseteq \mathrm{U}$, hence $\mathrm{F}(\mathrm{W}) \subseteq \mathrm{V}$.

Theorem 3.10. Let $\left(\mathrm{X}, \mu_{\mathrm{X}}\right)$ and $\left(\mathrm{Y}, \mu_{\mathrm{Y}}\right)$ be generalized topological spaces. The following are equivalent for a multifunction $\mathrm{F}: \mathrm{X} \rightarrow \mathrm{Y}:$

(1) $\mathrm{F}$ is lower $\alpha\left(\mu_{X}, \mu_{Y}\right)$-continuous;

(2) $\mathrm{F}^{-}(\mathrm{V}) \in \alpha\left(\mu_{\mathrm{X}}\right)$ for any $\mu_{Y^{-}}$open set $\mathrm{V}$ of $\mathrm{Y}$;

(3) $\mathrm{F}^{+}(\mathrm{M})$ is $\mu_{X}-\alpha$-closed in $X$ for any $\mu_{Y^{-}}$-closed set $M$ of $Y$;

(4) $i_{\sigma_{X}}\left(c_{\mu_{X}}\left(F^{+}(A)\right)\right) \subseteq F^{+}\left(c_{\mu_{Y}}(A)\right)$ for any subset $A$ of $Y$;

(5) $c_{\alpha_{X}}\left(F^{+}(A)\right) \subseteq F^{+}\left(c_{\mu_{Y}}(A)\right)$ for any subset $A$ of $Y$;

(6) $\mathrm{F}\left(\mathrm{c}_{\alpha_{X}}(\mathrm{~B})\right) \subseteq \mathrm{c}_{\mu_{Y}}(\mathrm{~F}(\mathrm{~B}))$ for any subset $\mathrm{B}$ of $\mathrm{X}$;

(7) $F\left(i_{\sigma_{X}}\left(c_{\mu_{X}}(B)\right)\right) \subseteq c_{\mu_{Y}}(F(B))$ for any subset $B$ of $X$;

(8) $F\left(c_{\mu_{X}}\left(i_{\mu_{X}}\left(c_{\mu_{X}}(B)\right)\right)\right) \subseteq c_{\mu_{Y}}(F(B))$ for any subset $B$ of $X$.

Proof. The proofs except for the following are similar to those of Theorem 3.9 and are thus omitted.

$(5) \Rightarrow(6)$ : Let $B$ be any subset of $X$. Since $B \subseteq F^{+}(F(B))$, we have

$$
\begin{aligned}
c_{\alpha_{X}}(\mathrm{~B}) & \subseteq \mathrm{c}_{\alpha_{X}}\left(\mathrm{~F}^{+}(\mathrm{F}(\mathrm{B}))\right) \\
& \subseteq \mathrm{F}^{+}\left(\mathrm{c}_{\mu_{Y}}(\mathrm{~F}(\mathrm{~B}))\right),
\end{aligned}
$$

and $\mathrm{F}\left(\mathrm{c}_{\alpha_{X}}(\mathrm{~B})\right) \subseteq \mathrm{c}_{\mu_{Y}}(\mathrm{~F}(\mathrm{~B}))$.

$(6) \Rightarrow(7)$ : This follows immediately from Lemma 3.8.

$(7) \Rightarrow(8)$ : This is obvious by Lemma 3.8 .

(8) $\Rightarrow(1)$ : Let $x \in X$ and $V$ be any $\mu_{Y}$-open set such that $F(x) \cap V \neq \emptyset$. Then $x \in F^{-}(V)$. We shall show that $\mathrm{F}^{-}(\mathrm{V}) \in \alpha\left(\mu_{\mathrm{X}}\right)$. By hypothesis, we have

$$
\begin{aligned}
\mathrm{F}\left(\mathrm{c}_{\mu_{X}}\left(i_{\mu_{X}}\left(c_{\mu_{X}}\left(F^{+}(\mathrm{Y}-\mathrm{V})\right)\right)\right)\right) & \subseteq \mathrm{c}_{\mu_{Y}}\left(\mathrm{~F}\left(\mathrm{~F}^{+}(\mathrm{Y}-\mathrm{V})\right)\right) \\
& \subseteq \mathrm{Y}-\mathrm{V},
\end{aligned}
$$

and hence $c_{\mu_{X}}\left(i_{\mu_{X}}\left(c_{\mu_{X}}\left(F^{+}(Y-V)\right)\right)\right) \subseteq F^{+}(Y-V)=X-F^{-}(V)$. Therefore, we obtain

$$
\mathrm{F}^{-}(\mathrm{V}) \subseteq i_{\mu_{X}}\left(c_{\mu_{X}}\left(i_{\mu_{X}}\left(F^{-}(\mathrm{V})\right)\right)\right)
$$

and hence $F^{-}(V) \in \alpha(\mu)$. Put $U=F^{-}(V)$. We have $x \in U \in \alpha\left(\mu_{X}\right)$ and $F(u) \cap V \neq \emptyset$ for every $u \in U$. Thus $F$ is lower $\alpha\left(\mu_{X}, \mu_{Y}\right)$-continuous. 
Definition 3.11 ([13]). A family $\mathcal{U}=\left\{\mathrm{U}_{\gamma}: \gamma \in \Gamma\right\}$ of sets in a generalized topological space $(X, \mu)$ is called $\mu$-locally finite, if for each $x \in X$ there exists $V \in \mu$ containing $x$ such that $V$ intersects at most finitely many members of $\mathcal{U}$.

Definition 3.12. A subset $A$ of a generalized topological space $\left(X, \mu_{X}\right)$ is said to be $\mu_{X}-\alpha$-paracompact, if every cover of $A$ by $\mu_{X}$-open sets of $X$ is refined by a cover of $A$ which consists of $\mu_{X}$-open sets of $X$ and is $\mu_{X}$-locally finite in $X$.

Definition 3.13. A subset $A$ of a generalized topological space $\left(X, \mu_{X}\right)$ is said to be $\mu_{X}-\alpha$-regular, if for each point $x \in A$ and each $\mu_{X}$-open set $U$ of $X$ containing $x$, there exists a $\mu_{X}$-open set $G$ of $X$ such that $x \in \mathrm{G} \subseteq \mathrm{c}_{\mu_{x}}(\mathrm{G}) \subseteq \mathrm{U}$.

Lemma 3.14. If $\mathrm{A}$ is a $\mu_{\mathrm{X}}-\alpha$-regular $\mu_{\mathrm{X}}$ - $\alpha$-paracompact subset of a quasi-topological space $\left(\mathrm{X}, \mu_{\mathrm{X}}\right)$ and $\mathrm{U}$ is a

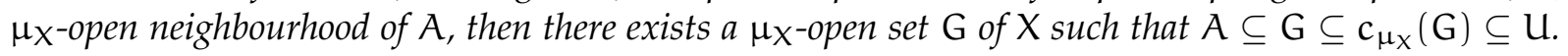

Definition 3.15. Let $\left(X, \mu_{X}\right)$ and $\left(Y, \mu_{Y}\right)$ be generalized topological spaces. A multifunction $F: X \rightarrow Y$ is said to be punctually $\mu$ - $\alpha$-paracompact (resp. punctually $\mu$ - $\alpha$-regular), if for each $x \in X, F(x)$ is $\mu_{Y}-\alpha$ paracompact (resp. $\mu_{\gamma}-\alpha$-regular).

By $c_{\alpha}(F): X \rightarrow Y$, we shall denote a multifunction defined as follows: $\left[c_{\alpha}(F)\right]^{+}(x)=c_{\alpha_{Y}}(F(x))$ for each point $x \in X$.

Lemma 3.16. Let $\left(\mathrm{X}, \mu_{\mathrm{X}}\right)$ be a generalized topological space and $\left(\mathrm{Y}, \mu_{\mathrm{Y}}\right)$ a quasi-topological space. If $\mathrm{F}: \mathrm{X} \rightarrow \mathrm{Y}$ is

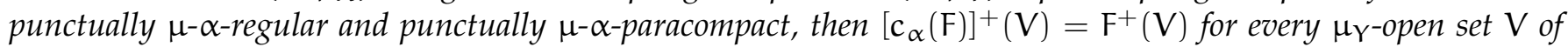
Y.

Proof. Let $\mathrm{V}$ be any $\mu_{\gamma^{-}}$-open set of $\mathrm{Y}$ and $\mathrm{x} \in\left[\mathrm{c}_{\alpha}(\mathrm{F})\right]^{+}(\mathrm{V})$. Then $\mathrm{c}_{\alpha_{\mathrm{Y}}}(\mathrm{F}(\mathrm{x})) \subseteq \mathrm{V}$ and hence $\mathrm{F}(\mathrm{x}) \subseteq \mathrm{V}$. Therefore, $x \in F^{+}(V)$ and hence $\left[c_{\alpha}(F)\right]^{+}(V) \subseteq F^{+}(V)$. Let $V$ be any $\mu_{Y^{-}}$open set of $Y$ and $x \in F^{+}(V)$. Then $F(x) \subseteq V$. Since $F(x)$ is $\mu_{Y}-\alpha$-regular and $\mu_{Y}-\alpha$-paracompact, by Lemma 3.14 there exists a $\mu_{Y}$-open set $G$ such that $\mathrm{F}(\mathrm{x}) \subseteq \mathrm{G} \subseteq \mathrm{c}_{\mu_{\gamma}}(\mathrm{G}) \subseteq \mathrm{V}$, hence $\mathrm{c}_{\alpha_{\gamma}}(\mathrm{F}(\mathrm{x})) \subseteq \mathrm{c}_{\mu_{\gamma}}(\mathrm{G}) \subseteq \mathrm{V}$. This shows that $\mathrm{x} \in\left[\mathrm{c}_{\alpha}(\mathrm{F})\right]^{+}(\mathrm{V})$ and hence $\mathrm{F}^{+}(\mathrm{V}) \subseteq\left[\mathrm{c}_{\alpha}(\mathrm{F})\right]^{+}(\mathrm{V})$. Consequently, we obtain $\left[\mathrm{c}_{\alpha}(\mathrm{F})\right]^{+}(\mathrm{V})=\mathrm{F}^{+}(\mathrm{V})$.

Theorem 3.17. Let $\left(\mathrm{X}, \mu_{\mathrm{X}}\right)$ be a generalized topological space and $\left(\mathrm{Y}, \mu_{\mathrm{Y}}\right)$ a quasi-topological space. Let $\mathrm{F}: \mathrm{X} \rightarrow \mathrm{Y}$ be punctually $\mu-\alpha$-regular and punctually $\mu$ - $\alpha$-paracompact. Then $\mathrm{F}$ is upper $\alpha\left(\mu_{\mathrm{X}}, \mu_{\mathrm{Y}}\right)$-continuous, if and only if $c_{\alpha}(\mathrm{F}): \mathrm{X} \rightarrow \mathrm{Y}$ is upper $\alpha\left(\mu_{\mathrm{X}}, \mu_{\mathrm{Y}}\right)$-continuous.

Proof. Suppose that $F$ is upper $\alpha\left(\mu_{X}, \mu_{Y}\right)$-continuous. Let $x \in X$ and $V$ be any $\mu_{Y}$-open set of $Y$ such that $c_{\mu}(F)(x) \subseteq V$. By Lemma 3.16, we have $x \in\left[c_{\mu}(F)\right]^{+}(V)=F^{+}(V)$. Since $F$ is upper $\alpha\left(\mu_{X}, \mu_{Y}\right)$-continuous, there exists $U \in \alpha\left(\mu_{X}\right)$ containing $x$ such that $F(U) \subseteq V$. Since $F(z)$ is $\mu_{Y}-\alpha$-regular and $\mu_{Y}$ - $\alpha$-paracompact for each $z \in \mathrm{U}$, by Lemma 3.14 there exists a $\mu$-open set $\mathrm{H}$ such that $\mathrm{F}(z) \subseteq \mathrm{H} \subseteq \mathrm{c}_{\mu_{\gamma}}(\mathrm{H}) \subseteq \mathrm{V}$. Therefore, we have $c_{\mu_{Y}}(F(z)) \subseteq c_{\mu_{Y}}(H) \subseteq V$ for each $z \in U$ and hence $c_{\mu}(F)(U) \subseteq V$. This shows that $c_{\mu}(F)$ is upper $\alpha\left(\mu_{X}, \mu_{Y}\right)$-continuous.

Conversely, suppose that $c_{\mu}(F): X \rightarrow Y$ is upper $\alpha\left(\mu_{X}, \mu_{Y}\right)$-continuous. Let $x \in X$ and $V$ be any $\mu_{Y^{-}}$ open set of $Y$ such that $F(x) \subseteq V$. By Lemma 3.16, we have $x \in F^{+}(V)=\left[c_{\mu}(F)\right]^{+}(V)$ and hence $c_{\mu}(F)(x) \subseteq$ $V$. Since $c_{\mu}(F)$ is upper $\alpha\left(\mu_{X}, \mu_{Y}\right)$-continuous, there exists $U \in \alpha\left(\mu_{X}, x\right)$ such that $c_{\mu}(F)(U) \subseteq V$, hence $\mathrm{F}(\mathrm{U}) \subseteq \mathrm{V}$. This shows that $\mathrm{F}$ is upper $\alpha\left(\mu_{X}, \mu_{Y}\right)$-continuous.

Lemma 3.18. Let $\left(\mathrm{X}, \mu_{\mathrm{X}}\right)$ and $\left(\mathrm{Y}, \mu_{\mathrm{Y}}\right)$ be generalized topological spaces. For a multifunction $\mathrm{F}: \mathrm{X} \rightarrow \mathrm{Y}$, it follows that for each $\mu_{\gamma^{-}} \alpha$-open set $\mathrm{V}$ of $\mathrm{Y}\left[\mathrm{c}_{\alpha}(\mathrm{F})\right]^{-}(\mathrm{V})=\mathrm{F}^{-}(\mathrm{V})$.

Proof. Suppose that $V$ is any $\mu_{Y}-\alpha$-open set of $Y$. Let $x \in\left[c_{\alpha}(F)\right]^{-}(V)$. Then $c_{\mu_{Y}}(F(x)) \cap V \neq \emptyset$ and hence $\mathrm{F}(\mathrm{x}) \cap \mathrm{V} \neq \emptyset$. Therefore, we obtain $x \in \mathrm{F}^{-}(\mathrm{V})$. This shows that $\left[\mathrm{c}_{\alpha}(\mathrm{F})\right]^{-}(\mathrm{V}) \subseteq \mathrm{F}^{-}(\mathrm{V})$. Let $x \in \mathrm{F}^{-}(\mathrm{V})$. Then we have $\emptyset \neq F(x) \cap V \subseteq c_{\alpha_{Y}}(F(x)) \cap V$ and hence $x \in\left[c_{\alpha}(F)\right]^{-}(V)$. This shows that $F^{-}(V) \subseteq\left[c_{\alpha}(F)\right]^{-}(V)$. Consequently, $\left[\mathrm{c}_{\alpha}(\mathrm{F})\right]^{-}(\mathrm{V})=\mathrm{F}^{-}(\mathrm{V})$. 
Theorem 3.19. Let $\left(\mathrm{X}, \mu_{\mathrm{X}}\right)$ and $\left(\mathrm{Y}, \mu_{\mathrm{Y}}\right)$ be generalized topological spaces. A multifunction $\mathrm{F}: \mathrm{X} \rightarrow \mathrm{Y}$ is lower $\alpha\left(\mu_{X}, \mu_{Y}\right)$-continuous, if and only if $c_{\alpha}(F): X \rightarrow Y$ is lower $\alpha\left(\mu_{X}, \mu_{Y}\right)$-continuous.

Proof. By utilizing Lemma 3.18, this can be proved similarly to that of Theorem 3.17.

For a multifunction $F: X \rightarrow Y$, the graph multifunction $G_{F}: X \rightarrow X \times Y$ is defined as follows: $\mathrm{G}_{\mathrm{F}}(\mathrm{x})=\{x\} \times \mathrm{F}(\mathrm{x})$ for every $\mathrm{x} \in \mathrm{X}$.

Lemma 3.20 ([19]). The following hold for a multifunction $\mathrm{F}: \mathrm{X} \rightarrow \mathrm{Y}$ :

(a) $\mathrm{G}_{\mathrm{F}}^{+}(\mathrm{A} \times \mathrm{B})=\mathrm{A} \cap \mathrm{F}^{+}(\mathrm{B})$;

(b) $G_{F}^{-}(A \times B)=A \cap F^{-}(B)$;

for any subsets $\mathrm{A} \subseteq \mathrm{X}$ and $\mathrm{B} \subseteq \mathrm{Y}$.

Definition 3.21 ([32]). A generalized topological space $(X, \mu)$ is called to be compact, if each cover of $X$ composed of elements of $\mu$ admits a finite subcover.

A subset $M$ of a generalized topological space $\left(X, \mu_{X}\right)$ is said to be $\mu_{X}$-compact, if every cover of $M$ by $\mu_{\mathrm{X}}$-open sets has a finite subcover.

Theorem 3.22 ([32]). A generalized topological space $(X, \mu)$ is compact, if and only if every family of $\mu$-closed subsets of $\mathrm{X}$ which has the finite intersection property has nonempty intersection.

Theorem 3.23. Let $\left(\mathrm{X}, \mu_{\mathrm{X}}\right)$ be a topological space and $\left(\mathrm{Y}, \mu_{\mathrm{Y}}\right)$ a generalized topological space. Let $\mathrm{F}: \mathrm{X} \rightarrow \mathrm{Y}$ be a multifunction such that $\mathrm{F}(\mathrm{x})$ is $\mu_{Y}$-compact for each $\mathrm{x} \in \mathrm{X}$. Then $\mathrm{F}$ is upper $\alpha\left(\mu_{X}, \mu_{Y}\right)$-continuous, if and only if $\mathrm{G}_{\mathrm{F}}: \mathrm{X} \rightarrow \mathrm{X} \times \mathrm{Y}$ is upper $\alpha\left(\mu_{\mathrm{X}}, \mu_{\mathrm{X} \times \mathrm{Y}}\right)$-continuous.

Proof. Suppose that $F: X \rightarrow Y$ is upper $\alpha\left(\mu_{X}, \mu_{Y}\right)$-continuous. Let $x \in X$ and $W$ be any $\mu_{X \times Y}$-open set of $X \times Y$ containing $G_{F}(x)$. For each $y \in F(x)$, there exists $\mu_{X}$-open set $U(y) \subseteq X$ and $\mu_{Y}$-open $V(y) \subseteq Y$ such that $(x, y) \in U(y) \times V(y) \subseteq W$. The family $\{V(y): y \in F(x)\}$ is a $\mu_{Y}$-open cover of $F(x)$ and there exist a finite number of points, say, $y_{1}, y_{2}, \ldots, y_{n}$ in $F(x)$ such that $F(x) \subseteq \cup\left\{V\left(y_{i}\right): 1 \leqslant i \leqslant n\right\}$. Set $\mathcal{G}=\cap\left\{U\left(y_{i}\right): 1 \leqslant i \leqslant n\right\}$ and $\mathcal{H}=\cup\left\{V\left(y_{i}\right): 1 \leqslant i \leqslant n\right\}$. Then $\mathcal{G}$ is $\mu_{X}$-open in $X$ and $\mathcal{H}$ is $\mu_{Y}$-open in $Y$ and $\{x\} \times F(x) \subseteq \mathcal{G} \times \mathcal{H} \subseteq W$. Since $F$ is upper $\alpha\left(\mu_{X}, \mu_{Y}\right)$-continuous, there exists $U_{0} \in \alpha\left(\mu_{X}\right)$ containing $x$ such that $\mathrm{F}\left(\mathrm{U}_{0}\right) \subseteq \mathcal{H}$. By Lemma 3.20, we have $\mathcal{G} \cap \mathrm{U}_{0} \subseteq \mathcal{G} \cap \mathrm{F}^{+}(\mathcal{H})=\mathrm{G}_{\mathrm{F}}^{+}(\mathcal{G} \times \mathcal{H}) \subseteq \mathrm{G}_{\mathrm{F}}^{+}(\mathrm{W})$. Therefore, we obtain $\mathcal{G} \cap \mathrm{U}_{0} \in \alpha\left(\mu_{\mathrm{X}}, \mathrm{x}\right)$ and $\mathrm{G}_{\mathrm{F}}\left(\mathcal{G} \cap \mathrm{U}_{0}\right) \subseteq \mathrm{W}$. This shows that $\mathrm{G}_{\mathrm{F}}$ is upper almost $\alpha\left(\mu_{\mathrm{X}}, \mu_{\mathrm{X} \times \mathrm{Y}}\right)$ continuous.

Conversely, suppose that $G_{F}: X \rightarrow X \times Y$ is upper $\alpha\left(\mu_{X}, \mu_{X \times Y}\right)$-continuous. Let $x \in X$ and $V$ be any $\mu_{Y}$-open set of $Y$ containing $F(x)$. Since $X \times V$ is $\mu_{X \times Y}$-open in $X \times Y$ and $G_{F}(x) \cap X \times V$, there exists $\mathrm{U} \in \alpha\left(\mu_{\mathrm{X}}\right)$ containing $x$ such that $\mathrm{G}_{\mathrm{F}}(\mathrm{U}) \cap \mathrm{X} \times \mathrm{V}$. Therefore, by Lemma 3.20, $\mathrm{U} \subseteq \mathrm{G}_{\mathrm{F}}^{+}(\mathrm{X} \times \mathrm{V})=\mathrm{F}^{+}(\mathrm{V})$ and hence $\mathrm{F}(\mathrm{U}) \subseteq \mathrm{V}$. This shows that $\mathrm{F}$ is upper $\alpha\left(\mu_{\mathrm{X}}, \mu_{Y}\right)$-continuous.

Theorem 3.24. Let $\left(\mathrm{X}, \mu_{\mathrm{X}}\right)$ and $\left(\mathrm{Y}, \mu_{\mathrm{Y}}\right)$ be generalized topological spaces. A multifunction $\mathrm{F}: \mathrm{X} \rightarrow \mathrm{Y}$ is lower $\alpha\left(\mu_{X}, \mu_{Y}\right)$-continuous, if and only if $\mathrm{G}_{\mathrm{F}}: \mathrm{X} \rightarrow \mathrm{X} \times \mathrm{Y}$ is lower $\alpha\left(\mu_{\mathrm{X}}, \mu_{\mathrm{X} \times \mathrm{Y}}\right)$-continuous.

Proof. Suppose that $F: X \rightarrow Y$ is lower $\alpha\left(\mu_{X}, \mu_{Y}\right)$-continuous. Let $x \in X$ and $W$ be any $\mu_{X \times Y}$-open set of $X \times Y$ such that $G_{F}(x) \cap W \neq \emptyset$. There exists $y \in F(x)$ such that $(x, y) \in W$ and hence $(x, y) \in U \times Y \subseteq W$ for some $\mu_{X}$-open set $U \subseteq X$ and $\mu_{Y}$-open set $V \subseteq Y$. Since $F(X) \cap V \neq \emptyset$, there exists $U_{0} \in \alpha\left(\mu_{X}\right)$ containing $x$ such that $\mathrm{F}(z) \cap \mathrm{V} \neq \emptyset$ for each $z \in \mathrm{U}_{0}$, hence $\mathrm{U}_{0} \subseteq \mathrm{F}^{-}(\mathrm{V})$. By Lemma 3.20, $\mathrm{U} \cap \mathrm{U}_{0} \subseteq \mathrm{U} \cap \mathrm{F}^{-}(\mathrm{V})=$ $\mathrm{G}_{\mathrm{F}}^{-}(\mathrm{U} \times \mathrm{V}) \subseteq \mathrm{G}_{\mathrm{F}}^{-}(\mathrm{W})$. Moreover, $x \in \mathrm{U} \cap \mathrm{U}_{0} \in \alpha\left(\mu_{\mathrm{X}}\right)$ and hence $\mathrm{G}_{\mathrm{F}}$ is lower $\alpha\left(\mu_{\mathrm{X}}, \mu_{\mathrm{X} \times \mathrm{Y}}\right)$-continuous.

Conversely, suppose that $G_{F}$ is upper $\alpha\left(\mu_{X}, \mu_{X \times Y}\right)$-continuous. Let $x \in X$ and $V$ be any $\mu_{Y}$-open set of $Y$ such that $F(X) \cap V \neq \emptyset$. Then $X \times V$ is $\mu_{X \times Y}$-open in $X \times Y$ and

$$
\begin{aligned}
\mathrm{G}_{\mathrm{F}}(\mathrm{x}) \cap \mathrm{X} \times \mathrm{V} & =(\{x\} \times \mathrm{F}(\mathrm{x})) \cap(\mathrm{X} \times \mathrm{V}) \\
& =\{x\} \times(\mathrm{F}(\mathrm{x}) \cap \mathrm{V}) \\
& \neq \emptyset .
\end{aligned}
$$


There exists $U \in \alpha\left(\mu_{X}\right)$ containing $x$ such that $G_{F}(z) \cap X \times V \neq \emptyset$ for each $z \in U$. By Lemma 3.20, we obtain $\mathrm{U} \subseteq \mathrm{G}_{\mathrm{F}}^{-}(\mathrm{X} \times \mathrm{V})=\mathrm{F}^{-}(\mathrm{V})$ and hence $\mathrm{F}(\mathrm{U}) \subseteq \mathrm{V}$. This shows that $\mathrm{F}$ is lower $\alpha\left(\mu_{\mathrm{X}}, \mu_{\mathrm{Y}}\right)$-continuous.

Definition 3.25. A generalized topological space $\left(X, \mu_{X}\right)$ is said to be $\mu_{X}-\alpha$-compact, if every cover of $X$ by $\mu_{X}-\alpha$-open sets has a finite subcover.

A subset $M$ of a generalized topological space $\left(X, \mu_{X}\right)$ is said to be $\mu_{X}-\alpha$-compact, if every cover of $X$ by $\mu_{X}-\alpha$-open sets has a finite subcover.

Theorem 3.26. Let $\left(X, \mu_{X}\right)$ and $\left(Y, \mu_{Y}\right)$ be generalized topological spaces. Let $\mathrm{F}: \mathrm{X} \rightarrow \mathrm{Y}$ be an upper $\alpha\left(\mu_{\mathrm{X}}, \mu_{\mathrm{Y}}\right)$ continuous surjective multifunction such that $\mathrm{F}(\mathrm{x})$ is $\mu_{\mathrm{Y}}$-compact for each $\mathrm{x} \in \mathrm{X}$. If $\left(\mathrm{X}, \mu_{\mathrm{X}}\right)$ is $\mu_{\mathrm{X}}$ - $\alpha$-compact, then $\left(\mathrm{Y}, \mu_{Y}\right)$ is $\mu_{Y}$-compact.

Proof. Let $\left\{V_{\gamma}: \gamma \in \Gamma\right\}$ be a $\mu_{Y}$-open cover of $Y$. For each $x \in X, F(x)$ is $\mu_{Y}$-compact and there exists a finite subset $\Gamma(x)$ of $\Gamma$ such that $F(x) \subseteq \cup\left\{V_{\gamma}: \gamma \in \Gamma(x)\right\}$. Set

$$
\mathrm{V}(\mathrm{x})=\cup\left\{\mathrm{V}_{\gamma}: \gamma \in \Gamma(\mathrm{x})\right\} .
$$

Since $F$ is upper $\alpha\left(\mu_{X}, \mu_{Y}\right)$-continuous, there exists $U(x) \in \alpha\left(\mu_{X}\right)$ containing $x$ such that $F(U(x)) \subseteq V(x)$. The family $\{\mathrm{U}(\mathrm{x}): x \in X\}$ is a $\mu_{X}-\alpha$-open cover of $X$ and there exist a finite number of points, say, $x_{1}$, $x_{2}, \cdots, x_{n}$ in $X$ such that $X=\cup\left\{U\left(x_{i}\right): 1 \leqslant i \leqslant n\right\}$. Therefore, we have $Y=F(X)=F\left(\cup_{i=1}^{n} U\left(x_{i}\right)\right)=$ $\cup_{i=1}^{n} F\left(U\left(x_{i}\right)\right) \subseteq \cup_{i=1}^{n} V\left(x_{i}\right)=\cup_{i=1}^{n} \cup_{\gamma \in \Gamma\left(x_{i}\right)} V_{\gamma}$. This shows that $\left(Y, \mu_{Y}\right)$ is $\mu_{Y}$-compact.

Definition 3.27 ([30]). A generalized topological space $(X, \mu)$ is said to be $\mu$-Hausdorff, if for any pair of distint points $x$ and $y$ of $X$, there exist disjoint $\mu$-open sets $U$ and $V$ of $X$ containing $x$ and $y$, respectively.

For a multifunction $F: X \rightarrow Y$, the graph $G(F)$ of $F$ is defined as follows: $G(F)=\{(x, y): x \in X$ and $y \in F(x)\}$.

Theorem 3.28. Let $\left(\mathrm{X}, \mu_{\mathrm{X}}\right)$ be a generalized topological space and $\left(\mathrm{Y}, \mu_{\mathrm{Y}}\right)$ a quasi-topological space. If $\mathrm{F}: \mathrm{X} \rightarrow \mathrm{Y}$ is an upper $\alpha\left(\mu_{X}, \mu_{Y}\right)$-continuous multifunction into a $\mu_{Y}$-Hausdorff space $\left(Y, \mu_{Y}\right)$ and $\mathrm{F}(\mathrm{X})$ is $\mu_{Y}$-compact for each

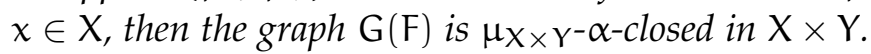

Proof. Let $(x, y) \in X \times Y-G(F)$. Then $y \in Y-F(x)$. For each $z \in F(x)$, there exist $\mu_{Y}$-open sets $V(z)$ and $\mathrm{W}(\mathrm{y})$ containing $z$ and $y$, respectively, such that $\mathrm{V}(z) \cap \mathrm{W}(\mathrm{y})=\emptyset$. The family $\{\mathrm{V}(z): z \in \mathrm{F}(\mathrm{x})\}$ is a $\mu_{Y}$-open cover of $F(x)$ and there exist a finite number of points in $F(x)$, say, $z_{1}, z_{2}, \cdots, z_{n}$ such that $\mathrm{F}(\mathrm{x}) \subseteq \cup\left\{\mathrm{V}\left(z_{\mathfrak{i}}\right): 1 \leqslant i \leqslant n\right\}$. Set $\mathcal{G}=\cup\left\{\mathrm{V}\left(z_{\mathfrak{i}}\right): 1 \leqslant i \leqslant n\right\}$ and $\mathcal{H}=\cap\left\{W\left(y_{\mathfrak{i}}\right): 1 \leqslant i \leqslant n\right\}$. Since $\mathrm{F}(\mathrm{x}) \subseteq \mathcal{G}$ and $\mathrm{F}$ is upper $\alpha\left(\mu_{X}, \mu_{Y}\right)$-continuous, there exists $\mathrm{U} \in \alpha\left(\mu_{X}\right)$ such that $\mathrm{x} \in \mathrm{U}$ and $\mathrm{F}(\mathrm{U}) \subseteq \mathcal{G}$. Therefore, we obtain $F(U) \cap \mathcal{H}=\emptyset$ and hence $(U \times \mathcal{H}) \cap G(F)=\emptyset$. Since $U \times \mathcal{H}$ is $\mu_{X \times Y^{-}} \alpha$-open in $X \times Y$ and $(x, y) \in U \times \mathcal{H},(x, y) \notin c_{\alpha_{X \times Y}}(G(F))$ and $G(F)$ is $\mu_{X \times Y}-\alpha$-closed in $X \times Y$.

In the following $(D,>)$ is a directed set, $\left(F_{\lambda}\right)$ is a net of multifunctions $F_{\lambda}: X \rightarrow Y, \lambda \in D$ where $F$ is multifunction on $\mathrm{X}$ into $\mathrm{Y}$.

Definition 3.29. Let $\left(F_{\lambda}\right)_{\lambda \in D}$ be a net of multifunctions on $X$ into $Y$. A multifunction $F^{\circledast}: X \rightarrow Y$ is defined as follows: for each $x \in X$,

$$
F^{\circledast}(x)=\left\{y \in Y \text { : for each } \mu_{Y} \text {-neighbourhood of } y \text { and each } \eta \in D \text { such that } \lambda>\eta \text { and } V \cap F_{\lambda}(x) \neq \emptyset\right\},
$$

is called the upper generalized topological limit of the net $\left(F_{\lambda}\right)$.

Definition 3.30. A net $\left(F_{\lambda}\right)_{\lambda \in D}$ is said to be equally upper $\alpha\left(\mu_{X}, \mu_{Y}\right)$-continuous at $x_{0} \in X$, if for every $\mu_{X}$-open set $V_{\lambda}$ containing $F_{\lambda}\left(x_{0}\right)$ there exists a $\mu_{X}$ - $\alpha$-open set $U$ containing $x_{0}$ such that $F_{\lambda}(U) \subseteq V_{\lambda}$ for all $\lambda \in$ D. 
Theorem 3.31. Let $\left(\mathrm{F}_{\lambda}\right)_{\lambda \in \mathrm{D}}$ be a net of multifunctions from a generalized topological space $\left(\mathrm{X}, \mu_{\mathrm{X}}\right)$ into a $\mu_{\mathrm{Y}^{-}}$ compact generalized topological space $\left(\mathrm{Y}, \mu_{\mathrm{Y}}\right)$. If the following are satisfied:

(a) $\cap\left\{\left(Y-F_{\eta}(x)\right): \eta>\lambda\right\} \in \mu_{Y}$, for each $\lambda \in D$ and each $x \in X$;

(b) $\left(F_{\lambda}\right)$ is equally upper $\alpha\left(\mu_{X}, \mu_{Y}\right)$ - continuous on $X$;

then $\mathrm{F}^{\circledast}$ is upper $\alpha\left(\mu_{\mathrm{X}}, \mu_{\mathrm{Y}}\right)$-continuous on $\mathrm{X}$.

Proof. It is known that $F^{\circledast}(x)=\cap\left\{c_{\mu_{\gamma}}\left(\cup\left\{F_{\eta}(x): \eta>\lambda\right\}\right): \lambda \in D\right\}$. From (a), we have

$$
\mathrm{F}^{\circledast}(x)=\cap\left\{\left[\cup\left\{\mathrm{F}_{\eta}(x): \eta>\lambda\right\}\right]: \lambda \in \mathrm{D}\right\} .
$$

Since the net $\left(\cup\left\{F_{\eta}(x): \eta>\lambda\right\}\right)_{\lambda \in D}$ is a family of $\mu_{Y}$-closed sets having the finite intersection property and $Y$ is $\mu_{Y}$-compact, it follows that $F^{\circledast}(x) \neq \emptyset$ for each $x \in X$. Now, let $x_{0} \in X$ and let $V \in \mu_{Y}$ such that $\mathrm{V} \neq \mathrm{Y}$ and $\mathrm{F}^{\circledast}\left(\mathrm{x}_{0}\right) \subseteq \mathrm{V}$. Then $\mathrm{F}^{\circledast}\left(\mathrm{x}_{0}\right) \cap(\mathrm{Y}-\mathrm{V})=\emptyset, \mathrm{F}^{\circledast}\left(\mathrm{x}_{0}\right) \neq \emptyset$ and $\mathrm{Y}-\mathrm{V} \neq \emptyset$. It results that $\cap\left\{\left[\cup\left\{\mathrm{F}_{\eta}(\mathrm{x}):\right.\right.\right.$ $\eta>\lambda\}]: \lambda \in D\} \cap(Y-V)=\emptyset$ and hence $\cap\left\{\left[\cup\left\{F_{\eta}\left(x_{0}\right) \cap(Y-V): \eta>\lambda\right\}\right]: \lambda \in D\right\} \cap(Y-V)=\emptyset$. Since $Y$ is $\mu_{Y}$-compact and the family $\left\{\left[\cup\left\{F_{\eta}\left(x_{0}\right) \cap(Y-V): \eta>\lambda\right\}\right]: \lambda \in D\right\}$ is a family of $\mu_{Y}$-closed sets with the empty intersection, there exists $\lambda \in D$ such that for each $\eta \in D$ with $\eta>\lambda$ we have $F\left(x_{0}\right) \cap(Y-V)=\emptyset$, hence $F_{\eta}\left(x_{0}\right) \subseteq V$. Since the net $\left(F_{\lambda}\right)_{\lambda \in D}$ is equally upper $\alpha\left(\mu_{X}, \mu_{Y}\right)$-continuous on $X$, it results that there exists a $\mu_{X}-\alpha$-open set $U$ containing $x_{0}$ such that $F_{\eta}(U) \subseteq V$ for each $\eta>\lambda$, hence $F_{\eta}(x) \cap(Y-V)=\emptyset$ for each $x \in U$. Then, we have $\cup\left\{F_{\eta}(x) \cap(Y-V): \eta>\lambda\right\}=\emptyset$, hence $\cap\left\{\left[\cup\left\{F_{\eta}(x): \eta>\lambda\right\}\right]: \lambda \in D\right\} \cap(Y-V)=\emptyset$. This implies that $F \circledast(U) \subseteq V$. If $V=Y$, then it is clear that for each $\mu_{X}-\alpha$-open set $U$ containing $x_{0}$ we have $F^{\circledast}(U) \subseteq V$. Hence, $F^{\circledast}$ is upper $\alpha\left(\mu_{X}, \mu_{Y}\right)$-continuous at $x_{0}$. Since $x_{0}$ is arbitrary, so the proof is complete.

Definition 3.32. The $\mu_{X}-\alpha$-frontier of a subset $A$ of a generalized topological space $\left(X, \mu_{X}\right)$, denoted by $\mu_{X}-\alpha-\operatorname{Fr}(A)$, is defined by $\mu_{X}-\alpha-\operatorname{Fr}(A)=c_{\alpha_{X}}(A) \cap c_{\alpha_{X}}(X-A)=c_{\alpha_{X}}(A)-i_{\alpha_{X}}(A)$.

Theorem 3.33. Let $\left(X, \mu_{X}\right)$ and $\left(Y, \mu_{Y}\right)$ be generalized topological spaces. The set of all points of $X$ at which a multifunction $\mathrm{F}: \mathrm{X} \rightarrow \mathrm{Y}$ is not upper (resp. lower) $\alpha\left(\mu_{\mathrm{X}}, \mu_{\mathrm{Y}}\right)$-continuous is identical with the union of the $\mu_{\mathrm{X}}-\alpha$-frontier of the upper (resp. lower) inverse images of $\mu_{\mathrm{X}}$-open sets containing (resp. meeting) $\mathrm{F}(\mathrm{X})$.

Proof. We prove only the case for upper $\alpha\left(\mu_{X}, \mu_{Y}\right)$-continuous because the case for lower $\alpha\left(\mu_{X}, \mu_{Y}\right)$ continuous is similarly shown. Let $x \in X$ at which $F$ is not upper $\alpha\left(\mu_{X}, \mu_{Y}\right)$-continuous. There exists a $\mu_{X}$-open set $V$ of $Y$ containing $F(x)$ such that $U \cap\left(X-F^{+}(V)\right) \neq \emptyset$ for every $U \in \alpha\left(\mu_{X}, x\right)$. Therefore, we have $x \in c_{\mu_{X}}\left(X-F^{+}(V)\right)=X-i_{\mu_{X}}\left(F^{+}(V)\right)$ and $x \in F^{+}(V)$. Hence, we obtain $x \in \mu_{X}-\alpha-F r(A)$.

Conversely, suppose that $V$ is $\mu_{X}$-open set of $Y$ containing $F(x)$ such that $x \in \mu_{X}-\alpha-\operatorname{Fr}(A)$. If $F$ is upper $\alpha\left(\mu_{X}, \mu_{Y}\right)$-continuous at $x$, there exists $U \in \alpha\left(\mu_{X}, x\right)$ such that $U \subseteq F^{+}(V)$. This implies that $x \in i_{\alpha_{X}}\left(F^{+}(V)\right)$. This is a contradiction and hence $F$ is not upper $\alpha\left(\mu_{X}, \mu_{Y}\right)$-continuous at $x$.

Definition 3.34 ([31]). A generalized topological space $(X, \mu)$ is said to be connected (called $\mu$-connected in [6]), if there are no nonempty disjoint sets $U, V \in \mu$ such that $U \cup V=X$.

Definition 3.35 ([31]). A generalized topological space $(X, \mu)$ is said to be $\alpha$-connected, if $(X, \alpha(\mu))$ is connected.

Definition 3.36. Let $\left(X, \mu_{X}\right)$ and $\left(Y, \mu_{Y}\right)$ be generalized topological spaces. A multifunction $F: X \rightarrow Y$ is said to be punctually $\mu$-connected, if for each $x \in X, F(x)$ is $\mu_{Y}$-connected.

Theorem 3.37. Let $\mathrm{F}$ be a multifunction from an $\alpha$-connected generalized topological space $\left(\mathrm{X}, \mu_{\mathrm{X}}\right)$ into a generalized topological space $\left(\mathrm{Y}, \mu_{\mathrm{Y}}\right)$ such that $\mathrm{F}$ is punctually $\mu$-connected. If $\mathrm{F}$ is upper $\alpha\left(\mu_{\mathrm{X}}, \mu_{\mathrm{Y}}\right)$-continuous, then $\left(Y, \mu_{Y}\right)$ is $\mu_{Y}$-connected.

Proof. Suppose that $\left(\mathrm{Y}, \mu_{\mathrm{Y}}\right)$ is not $\mu_{\mathrm{Y}}$-connected and let $\mathrm{Y}=\mathrm{U} \cup \mathrm{V}$ be a partition of $\mathrm{Y}$. Then both $\mathrm{U}$ and $V$ are $\mu_{Y}$-open and $\mu_{Y}$-closed subsets of $Y$. Since $F$ is upper $\alpha\left(\mu_{X}, \mu_{Y}\right)$-continuous, $F^{+}(U)$ and $F^{+}(U)$ are 


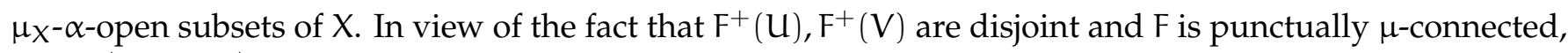
$X=F^{+}(U) \cup F^{+}(V)$ is a partition of $X$. This is contrary to the $\alpha$-connectedness of $\left(X, \mu_{X}\right)$. Hence, it is obtained that $\left(Y, \mu_{Y}\right)$ is $\mu_{Y}$-connected.

Definition 3.38 ([29]). A generalized topological space $(X, \mu)$ is said to be $\mu$-regular, if for each $\mu$-closed set $F$ of $X$ not containing $x$, there exist disjoint $\mu$-open sets $U$ and $V$ such that $x \in U$ and $F \subseteq V$.

Definition 3.39. A generalized topological space $\left(X, \mu_{X}\right)$ is said to be $\mu_{X}-\alpha-T_{2}$, if for any pair of distinct

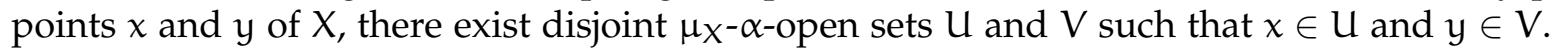

Definition 3.40. Let $\left(X, \mu_{X}\right)$ and $\left(Y, \mu_{Y}\right)$ be generalized topological spaces. A multifunction $F: X \rightarrow Y$ is said to be punctually $\mu$-closed, if for each $x \in X, F(x)$ is $\mu_{Y}$-closed.

Theorem 3.41. Let $\mathrm{F}: \mathrm{X} \rightarrow \mathrm{Y}$ be an upper $\alpha\left(\mu_{\mathrm{X}}, \mu_{\mathrm{Y}}\right)$-continuous multifunction and punctually $\mu$-closed from a generalized topological space $\left(\mathrm{X}, \mu_{\mathrm{X}}\right)$ to a $\mu_{\mathrm{Y}}$-normal generalized topological space $\left(\mathrm{Y}, \mu_{\mathrm{Y}}\right)$ and let $\mathrm{F}(\mathrm{X}) \cap \mathrm{F}(\mathrm{y})=\emptyset$ for each distinct pair $\mathrm{x}, \mathrm{y} \in \mathrm{X}$. Then $\left(\mathrm{X}, \mu_{\mathrm{X}}\right)$ is $\mu_{\mathrm{X}}-\alpha-\mathrm{T}_{2}$.

Proof. Let $x$ and $y$ be any two distinct points in $X$. Then we have $F(x) \cap F(y)=\emptyset$. Since $\left(Y, \mu_{Y}\right)$ is a $\mu_{Y}$-normal space, it follows that there exist disjoint $\mu_{Y}$-open sets $U$ and $V$ containing $F(x)$ and $F(y)$, respectively. Therefore, $F^{+}(U)$ and $F^{+}(U)$ are disjoint $\mu_{X-\alpha}-\alpha$-open sets containing $x$ and $y$, respectively. Thus, it is obtained that $\left(X, \mu_{X}\right)$ is $\mu_{X}-\alpha-T_{2}$.

\section{Acknowledgment}

This research was financially supported by the Faculty of Science, Mahasarakham University.

\section{References}

[1] M. E. Abd El-Monsef, S. N. El-Deeb, R. A. Mahmoud, $\beta$-open sets and $\beta$-continuous mapping, Bull. Fac. Sci. Assiut Univ. A, 12 (1983), 77-90. 1

[2] M. E. Abd El-Monsef, A. A. Nasef, On multifunctions, Chaos Solitons Fractals, 12 (2011), 2387-2394. 1

[3] D. Andrijević, Semi-preopen sets, Math. Vesnik, 38 (1986), 24-32. 1

[4] C. Boonpok, On upper and lower $\beta\left(\mu_{X}, \mu_{Y}\right)$-continuous multifunctions, Int. J. Math. Math. Sci., 2012 (2012), 17 pages. 2.3

[5] Á. Császár, Generalized topology, generalized continuity, Acta Math. Hungar., 96 (2002), 351-357. 1, 2

[6] Á. Császár, $\gamma$-connected sets, Acta Math. Hungar., 101 (2003), 273-279. 3.34

[7] Á. Császár, Extremally disconnected generalized topologies, Ann. Univ. Sci. Budapest. Eötvös Sect. Math., 47 (2004), 91-96. 2

[8] Á. Császár, Generalized open sets in generalized topologies, Acta Math. Hungar., 106 (2005), 53-66. 2

[9] Á. Császár, Further remarks on the formula for $\gamma$-interior, Acta Math. Hungar., 113 (2006), 325-332. 2

[10] Á. Császár, Modification of generalized topologies via hereditary classes, Acta Math. Hungar., 115 (2007), 29-36. 2

[11] Á. Császár, $\delta$ - and $\theta$-modifications of generalized topologies, Acta Math. Hungar., 120 (2008), 275-279. 2

[12] Á. Császár, Product of generalized topologies, Acta Math. Hungar., 123 (2009), 127-132. 2, 2.1

[13] A. Deb Ray, R. Bhowmick, $\mu$-paracompact and $\mathrm{g}_{\mu}$-paracompact generalized topological spaces, Hacet. J. Math. Stat., 45 (2016), 447-453. 3.11

[14] A. Kanibir, I. L. Reilly, Generalized continuity for multifunctions, Acta Math. Hungar., 122 (2009), 283-292. 1

[15] N. Levine, Semi-open sets and semi-continuity in topological spaces, Amer. Math. Monthly, 70 (1963), 36-41. 1

[16] M. S. Mashhour, M. E. Abd El-Monsef, S. N. El-Deeb, On precontinuous and weak precontinuous mappings, Proc. Math. Phys. Soc. Egypt, 53 (1982), 47-53. 1

[17] T. Neubrunn, Strongly quasi-continuous multivalued mappings, General topology and its relations to modern analysis and algebra, VI, Prague, (1986), 351-359, Res. Exp. Math., Heldermann, Berlin, (1988). 1

[18] O. Njàstad, On some classes of nearly open sets, Pacific J. Math., 15 (1965), 961-970. 1

[19] T. Noiri, V. Popa, Almost weakly continuous multifunctions, Demonstratio Math., 26 (1993), 363-380. 3.20

[20] T. Noiri, V. Popa, On upper and lower almost $\beta$-continuous multifunctions, Acta Math. Hungar., 82 (1999), 57-73. 1

[21] J. H. Park, B. Y. Lee, M. J. Son, On upper and lower $\delta$-precontinuous multifunctions, Chaos Solitons Fractals, 19 (2004), 1231-1237. 1

[22] V. I. Ponomarev, Properties of topological spaces preserved under multivalued continuous mapping on compacta, Amer. Math. Soc. Translations, 38 (1964), 441-446. 1 
[23] V. Popa, Sur certaines formes faibles de continuité pour les multifonctions, (French) [[Some weak forms of continuity for multifunctions]] Rev. Roumaine Math. Pures Appl., 30 (1985), 539-546. 1

[24] V. Popa, Some properties of H-almost continuous multifunctions, Problemy Mat., 10 (1990), 9-26. 1

[25] V. Popa, T. Noiri, On upper and lower $\alpha$-continuous multifunctions, Math. Slovaca, 43 (1993), 477-491. 1

[26] V. Popa, T. Noiri, On upper and lower almost $\alpha$-continuous multifunctions, Demonstratio Math., 29 (1996), $381-396$.

[27] V. Popa, T. Noiri, On upper and lower $\beta$-continuous multifunctions, Real Anal. Exchange, 22 (1996/97), 362-376.

[28] V. Popa, T. Noiri, On upper and lower weakly $\alpha$-continuous multifunctions, Novi Sad J. Math., 32 (2002), 7-24. 1

[29] B. Roy, On a type of generalized open sets, Appl. Gen. Topol., 12 (2011), 163-173. 3.38

[30] M. S. Sarsak, Weak separation axioms in generalized topological spaces, Acta Math. Hungar., 131 (2011), 110-121. 3.27

[31] R.-X. Shen, A note on generalized connectedness, Acta Math. Hungar., 122 (2009), 231-235. 3.34, 3.35

[32] R.-X. Shen, Remarks on products of generalized topologies, Acta Math. Hungar., 124 (2009), 363-369. 2.2, 3.21, 3.22 